\title{
Fuzzy Based Clustering ANd Energy EFficient ROUTING FOR UNDERWATER Wireless SENSOR NETWORKS
}

\author{
Sihem Souiki ${ }^{1}$, Mourad Hadjila ${ }^{1}$ and Mohammed Feham ${ }^{1}$ \\ ${ }^{1}$ Department of Telecommunication, Tlemcen University, Algeria.
}

\begin{abstract}
Underwater Wireless Sensor Network (UWSN) is a particular kind of sensor networks which is characterized by using acoustic channels for communication. UWSN is challenged by great issues specially the energy supply of sensor node which can be wasted rapidly by several factors. The most proposed routing protocols for terrestrial sensor networks are not adequate for UWSN, thus new design of routing protocols must be adapted to this constrain. In this paper we propose two new clustering algorithms based on Fuzzy C-Means mechanisms. In the first proposition, the cluster head is elected initially based on the closeness to the center of the cluster, then the node having the higher residual energy elects itself as a cluster head. All non-cluster head nodes transmit sensed data to the cluster head. This latter performs data aggregation and transmits the data directly to the base station. The second algorithm uses the same principle in forming clusters and electing cluster heads but operates in multi-hop mode to forward data from cluster heads to the underwater sink (uw-sink). Furthermore the two proposed algorithms are tested for static and dynamic deployment. Simulation results demonstrate the effectiveness of the proposed algorithms resulting in an extension of the network lifetime.
\end{abstract}

\section{KEYWORDS}

UWSN, Routing, Clustering, Fuzzy C-Means, Energy efficiency

\section{INTRODUCTION}

In recent years, underwater wireless sensor network has emerged as a powerful technique in order to discover and exploit this harsh environment. As over $70 \%$ of the earth's surface is covered by water, it is advantageous to deploy underwater sensor networks to support several categories of applications such as oceanographic data collection, pollution monitoring, offshore exploration, disaster prevention, assisted navigation and tactical surveillance applications [1]. To make these applications viable, there is a need to enable underwater communications among underwater devices. The underwater communication may include the transmission of information in three forms (sound, electromagnetic (EM), or optical waves). Each of these techniques has advantages and drawbacks. Electromagnetic signals deliver very poor performance underwater, providing transmission ranges of only a few meters at the typical RF sensor transmission power. Optical communication for underwater sensor networks using light waves has also been investigated; however these methods either require high precision or high power if the distances between sensor nodes are large. Consequently, acoustic networks enabled by sound waves become ideal alternatives since acoustic signals propagate well through water and require much less power than $\mathrm{RF}$ and light signals for the same communication range [2]. 
An underwater sensor network is usually formed by several autonomous and individual sensor nodes used to collect and forward data to the uw-sink. The most important challenges of deploying such a network are the cost, the computational power, the memory, the communication range and most of all the limited battery resources of each sensor node. The lifetime of UWSN is largely restricted because the number of sensor nodes that stop working due to the energy wastage increases with a deployment time. Then the high energy consumption is particularly significant defy for researchers to achieve long operating time without affecting system performance.

Generally routing is the backbone for any network, and routing protocols are considered to be in charge for discovering and maintaining the routes. Most of the proposed protocols for terrestrial sensor networks cannot be immediately used in UWSN owing to the continuous exchange of overhead messages applied (proactive ad hoc routing) or the route discovery process based on the flooding technique (reactive ad hoc routing) although the major protocols are designed for a stationary deployment, thus these solutions are ineffective in large scale UWSN because they exhaust energy and bandwidth resources. Therefore new energy efficient protocols must be designed for UWSN.

In this paper we present a hierarchical fuzzy based energy efficient routing algorithms where the clusters are formed by the Fuzzy C-Means method. The nodes are deployed randomly in three dimensional environments. The first proposed algorithm employs a single hop transmission between cluster heads and the uw-sink. Whereas the second proposition uses the multihop transmission between cluster heads and uw-sink; both algorithms are simulated for static and dynamic topologies.

The remainder of the paper is organized as follows. In section 2, a related work on hierarchical routing in both terrestrial and underwater wireless sensor networks are presented. Section 3 gives a detail description of our routing algorithms simulated in static and dynamic topologies. Section 4 shows the simulations results. Finally, the last section concludes the paper.

\section{RELATED WORKS}

Energy saving is a primordial issue in UWSNs because sensor nodes are supplied by batteries, which are difficult to replace or recharge in hostile underwater environments. Designing robust, scalable and energy aware routing protocols in this kind of networks is a fundamental research challenge. In the ground based wireless sensor networks, numerous routing protocols have been developed which can be divided into following classes according to deployment: flat, geographical, and hierarchal routing. In a flat topology, all nodes perform the same tasks and have the same functionalities in the network. Data transmission is performed by flooding in hop by hop manner. Flooding [3], Gossiping [4] and SPIN [5] are example of flat routing protocols. The second class is based on the position information of each node to determine forwarding path. The typical geographic routing protocols in WSNs include [6,7].Owing to satisfy the scalability aim and extending network lifetime in WSN, grouping nodes into clusters has been widely adopted by the research community. The hierarchical routing protocols involve cluster-based structure of the sensor nodes. Generally, each cluster constitutes a leader referred to as cluster head $(\mathrm{CH})$ usually performs the special tasks (fusion and aggregation) and other member nodes (collection of data and monitoring). The first clustering routing protocol proposed for WSNs includes Low-energy Adaptive Clustering Hierarchy (LEACH) [8]. The key idea of this protocol is to select randomly a set of sensor nodes as cluster head and rotate this task to uniformly distribute the energy load among the nodes in the network. There are two phases of LEACH protocol: The setup and steady phases. Firstly, in the setup phase clusters are formed and the cluster head $(\mathrm{CH})$ selection is performed by the member nodes. Secondly the cluster head $(\mathrm{CH})$ compress the gathered data 
from diverse nodes that belong to the respective cluster. Then the cluster head forwards aggregated data to the base station by single hop communication. Multiple variants of LEACH protocol are proposed to overcome some drawbacks of this protocol such as: LEACH-C [9], MRLEACH [10] and HEED [11].

However, these protocols are not appropriate for UWSNs because they assume that the sensor network is stationary and they are not well adapted to the intrinsic properties of underwater environments, such as long propagation delays, low data rates and difficulty of synchronization.

On the other hand, some hierarchical routing protocols have been designed for UWSN such as Ducs [12], Mccp [13] and HydroCast [14].

DUCS protocol is designed for long-term non-time critical applications where the sensor nodes are grouped into clusters using a distributed algorithm. The protocol operates in two stages: the first stage containing the clusters formation and the selection of the cluster head based on the remaining energy. In addition a randomized rotation of $\mathrm{CH}$ is performed among different nodes within a cluster in order to alleviate fast draining of the sensor node energy. In the second stage the data are transmitted to the sink using multi-hop routing through other cluster heads.

In MCCP protocol (Minimum Cost Clustering Protocol), the clusters are created based on a cost metric. The cost metric is calculated on the basis of three important parameters: (1) the total energy consumption of the cluster members for sending data to the cluster head; (2) the residual energy of the cluster head and its cluster members; and (3) the relative location between the cluster head and the uw-sink. The proposed protocol selects a set of non-overlapping clusters from all potential clusters based on the cost metric affected to each potential cluster and attempts to reduce the cost of the selected clusters. MCCP can adapt geographical cluster head distribution to the traffic pattern in the network and thus avoid the formation of hot spots around the uw-sink. It can also balance the traffic load between cluster heads and cluster members through periodical re-clustering the sensor nodes in the network.

The global idea of HydroCast is based on a routing decision which is made after comparing the local pressure or depth information, such that data packets are greedily forwarded towards a node with the lowest pressure level among the neighbor nodes. In HydroCast scheme, each local maximum node maintains a recovery route towards a neighboring node with higher depth than itself. After one or several forwarding's through local maxima, a data packet can be routed out of the void region and can be switched back to the greedy mode.

\section{THE PROPOSED ALGORITHMS}

In the following, we briefly introduce the basic theory of Fuzzy C-Means (FCM) used in cluster formation of our propositions, and then we give a detailed description of the proposed approaches.

\subsection{Basic theory of Fuzzy C-Means}

Fuzzy C-Means clustering algorithm [15], is a kind of clustering algorithm using membership to describe the possibility of cluster. However FCM is a local optimization algorithm, which is very sensitive to initialization and gets into the local minimum value easily.

The finite vectors $\mathrm{x}_{\mathrm{i}}(\mathrm{i}=1,2, \ldots, \mathrm{n})$ are divided into $\mathrm{c}(1<\mathrm{c}<\mathrm{n})$ classes, and the clustering center of each class is solved to make membership minimum as the non-similarity index. 
The objective function can be defined as follows:

$$
J\left(U, c_{1}, c_{2} \ldots c_{c}\right)=\sum_{i=1}^{c} \sum_{j}^{n} U_{i j}^{m} d_{i j}^{2}
$$

Where $U_{i j}$ is the membership of the group, $c_{i}$ is the clustering center; $d_{i j}$ is the special distance from vector $c_{i}$ to $x_{j} . m$ is the weighted index.

The steps of algorithm are as the following:

- Initializing the membership matrix $U$ to make it satisfy the following formula.

$$
\sum_{i=1}^{c} U_{i j}=1, \forall j=1, \ldots, n
$$

- Calculating the clustering center using the following formula.

$$
\mathrm{C}_{\mathrm{i}}=\frac{\sum_{\mathrm{j}=1}^{\mathrm{n}} \mathrm{U}_{\mathrm{ij}}^{\mathrm{m}} \mathrm{x}_{\mathrm{j}}}{\sum_{\mathrm{j}=1}^{\mathrm{n}} \mathrm{U}_{\mathrm{ij}}^{\mathrm{m}}}
$$

- Calculating the objective function according to the formula (1). If the objective function is less than a threshold or the relative value function change value last time is less than a threshold, the algorithm stops.

- Updating the matrix by the following formula and returning to step2.

$$
\mathrm{U}_{\mathrm{ij}}=\sum_{\mathrm{k}=1}^{\mathrm{c}}\left(\frac{\mathrm{d}_{\mathrm{ij}}}{\mathrm{d}_{\mathrm{kj}}}\right)^{-2 / m-1}
$$

\subsection{Single-Hop Fuzzy based Energy Efficient Routing algorithm for UWSN (SH- FEER):}

SH-FEER is a fuzzy based energy efficient algorithm where clusters are formed by using the Fuzzy C-Means method. We suppose that underwater sensor nodes always have data to be sent to the sink and the set of nodes have the same amount of energy [16].

We assume in this approach that the nodes organize themselves inside clusters randomly with unequal sizes and one node is selected as a cluster-head for each cluster. All non-cluster head nodes forward their data to their cluster head via a single hop; the cluster-head node receives data from all cluster members, performs signal processing functions on the data (e.g. aggregation) and transmits the data to the sink using single-hop routing. The cluster heads are responsible for coordination among nodes within their clusters (intra-cluster coordination) and communication between each other (inter-cluster coordination).

SH-FEER incorporates rotation of the cluster-head among the sensors to avoid rapid draining of the batteries of specific underwater sensors. In this way, the energy consumption is distributed. The operation mode of SH-FEER is composed to three phases: clusters formation during the first 
step and secondly the cluster head are selected. Initially, the closest node to the center is selected as cluster head and in the next rounds the selection is based on the residual energy of each node; the third step is the transmission of data towards the sink.

The algorithm of this first proposition is mentioned as follows:

Step 1: Clusters formation

Apply FCM algorithm to form clusters.

- Each cluster K(i) contains a number of nodes, $\mathrm{i}=1, \ldots, \mathrm{N}$

- Initially all nodes have the same amount of energy.

Step 2: Cluster head selection

$\operatorname{maxE}=\mathrm{zeros}(1, \mathrm{~N})$;

maxE is a row vector contains $\mathrm{N}$ zeros

$\mathrm{R} \_$max: maximum number of rounds

TE: total energy of network

while $\left(\mathrm{R} \leq \mathrm{R} \_\right.$max $\left.\| \mathrm{TE}>0\right)$

for $\mathrm{i}=1$ to $\mathrm{N}$ do

if $\mathrm{R}==1$

-calculate the distance $\mathrm{d}$ (nodei , center) // between nodei and center of cluster.

-Assign ICH (i) of the cluster in which d(nodei, center) is minimum.

else

for $\mathrm{j}=1$ to length( $\mathrm{k}(\mathrm{i}))$ do

ifmaxE(i)<k(i).E(j)

$\max E(i)=k(i) \cdot E(j)$

end if

end for

$\mathrm{CH}(\mathrm{i})=\operatorname{maxE}(\mathrm{i})$

end if

end for

Step 3: Data transmission

- Intra_cluster transmission

for $\mathrm{i}=1$ to $\mathrm{N}$ do

for $\mathrm{j}=1$ to length(k(i)) do

$\mathrm{k}(\mathrm{i}) . \mathrm{j}$ send data to $\mathrm{CH}(\mathrm{i})$

end for

end for

- Transmission from $\mathrm{CHs}$ to the uw-sink

for $\mathrm{i}=1$ to $\mathrm{N}$ do

$\mathrm{CH}(\mathrm{i})$ aggregates and forwards directly the data to uw-sink end for. 
International Journal of Computer Networks \& Communications (IJCNC) Vol.7, No.2, March 2015

\subsection{Multi-Hop Fuzzy based Energy Efficient Routing algorithm for UWSN (MH- FEER):}

MH-FEER mimics the first proposed algorithm; it uses the same two first phases of SH-FEER (clusters formation and cluster head selection). However the process of data forwarding toward the uw-sink is different by using multi-hop routing between cluster heads and uw-sink. The data are transferred through multiple cluster-heads in the direction of the uw-sink choosing the shortest path; this is repeated until it reaches the uw-sink.

Since the mode of clusters formation and the cluster heads selection is the same as the first algorithm, we present below only the pseudo-code relating to the data forwarding from the cluster heads to uw-sink.

Calculation of distances between $\mathrm{CHs}$ and distances between $\mathrm{CHs}$ and uw-sink

For $\mathrm{i}=1$ to $\mathrm{N}$

For $\mathrm{j}=1$ to $\mathrm{N}$ do

$$
\begin{gathered}
d(i, j)=\sqrt{\left(x_{i}-x_{j}\right)^{2}+\left(y_{i}-y_{j}\right)^{2}+\left(z_{i}-z_{j}\right)^{2}} \\
d_{u w-\operatorname{sink}}(i)=\sqrt{\left(x_{i}-x_{u w-s i n k}\right)^{2}+\left(y_{i}-y_{u w-\operatorname{sink}}\right)^{2}+\left(z_{i}-z_{u w-\operatorname{sink}}\right)^{2}}
\end{gathered}
$$

end for

for $\mathrm{i}=1$ to $\mathrm{N}$ do

ifd $_{\text {uw-sink (i) }}==$ Min $_{\text {duw-sink }}$

$\mathrm{CH}(\mathrm{i})$ sends directly to uw-sink

else

for $\mathrm{j}=1$ to $\mathrm{N}$ do

if $\left(\mathrm{i} \neq \mathrm{j} \& \& \mathrm{~d}(\mathrm{i}, \mathrm{j})==\min (\mathrm{i}) \& \& \mathrm{~d}_{\mathrm{uw}-\operatorname{sink}(\mathrm{i})}>\mathrm{d}_{\mathrm{uw}-\operatorname{sink}(\mathrm{j})}\right)$

$\mathrm{CH}(\mathrm{i})$ sends data to $\mathrm{CH}(\mathrm{j})$

end if

end for

end if

end for

\section{PERFORMANCE EVALUATION}

In this section, we evaluate the performance of the proposed algorithms through extensive simulations under Matlab environment. Firstly, we define the performance metrics and the simulation methodology, and then we present the energy model used. Moreover we evaluate how network parameters such as node density, node mobility affect the performance of the proposed algorithms. 
International Journal of Computer Networks \& Communications (IJCNC) Vol.7, No.2, March 2015

\subsection{Metrics and Methodology}

\subsubsection{Metrics}

In this paper two metrics are used to analyze the performance of the proposed algorithms: total energy consumption and the number of alive nodes

(i) Total energy consumption ET: is the sum of energy amount consumed by the all sensor nodes formed the networks

$$
\begin{aligned}
& \mathrm{E}(\mathrm{i})_{\text {consumed }}=\mathrm{E}(\mathrm{i})_{\text {initial }} \mathrm{E}(\mathrm{i})_{\text {residual }} \\
& \mathrm{ET}_{\text {consumed }}=\sum_{\mathrm{i}=1}^{\mathrm{N}} \mathrm{E}(\mathrm{i})_{\text {consumed }}
\end{aligned}
$$

(ii) Number of alive nodes: is the number of sensor nodes where the energy is different to 0 during (r) rounds.

\subsubsection{Simulation Methodology}

In our simulation we make some assumptions then we present the energy model used.

\section{- Assumptions}

- Sensor nodes as well as the uw-sink are stationary after being deployed in the field.

- The network is considered homogeneous and all of the sensor nodes have the same initial energy.

- Each sensor node knows its own geographical position.

- The underwater sink is not limited in terms of energy, memory and computational power.

- Underwater sink is located outside the area of the sensors nodes (at the surface).

- All nodes measure the environmental parameters at a fixed rate and send it periodically to the receiver nodes.

- Each sensor node can operate either in sensing mode to monitor the environment parameters and transmit to the underwater sink, cluster head (to compress and forward it to the uw-sink).

The Parameters setting of the simulation are shown in Table1

Table 1. Simulation parameters

\begin{tabular}{|l|l|}
\hline Parameters & Values \\
\hline Network size & $(100 * 100 * 100) \mathrm{m}^{3}$ \\
\hline Number of nodes & 100 \\
\hline Initial energy & $10 \mathrm{~J}$ \\
\hline Coordinate of the sink & $(50,50,150)$ \\
\hline
\end{tabular}

\section{- Energy model}

We use the same energy model as used in [17], which was proposed for underwater acoustic networks. According to this model, to achieve a power level $\mathrm{P}_{0}$ at a receiver at a distance $\mathrm{d}$, the transmitter power $\mathrm{E}_{\mathrm{tx}}(\mathrm{d})$ must be: 


$$
E_{t x}(d)=P_{0} \cdot d^{2} \cdot 10^{\frac{\alpha(f)}{10}}
$$

Where $\alpha(\mathrm{f})$, measured in $\mathrm{dB} / \mathrm{m}$, is a medium absorption coefficient depending on the frequency range of interest under given water temperature and salinity, $\alpha(f)$ is given by

$$
\alpha(\mathrm{f})=0.11 \frac{10^{-3} \mathrm{f}^{2}}{1+\mathrm{f}^{2}}+44 \frac{10^{-3} \mathrm{f}^{2}}{4100+\mathrm{f}^{2}}+2.75 \times 10^{-7} \mathrm{f}^{2}+3 \times 10^{-6}
$$

Where $\mathrm{f}$ is the carrier frequency for transmission in KHz. The reception power is assumed to $1 / 3$ th of the transmission power.

\subsection{Simulation1: static topology}

The figure 1 depicts the total energy consumption of the two proposed algorithms and a comparison is performed with the direct transmission. As shown in this figure we observe that the MH-FEER algorithm consumes less energy comparing with SH-FEER, this is due to the multi-hop routing used between cluster heads and the sink i.e. MH-FEER avoid the long distances transmission utilized in the SH-FEER between $\mathrm{CH}$ and the sink. Although this figure shows that the energy depletion of the two proposed algorithms is better than the direct transmission.
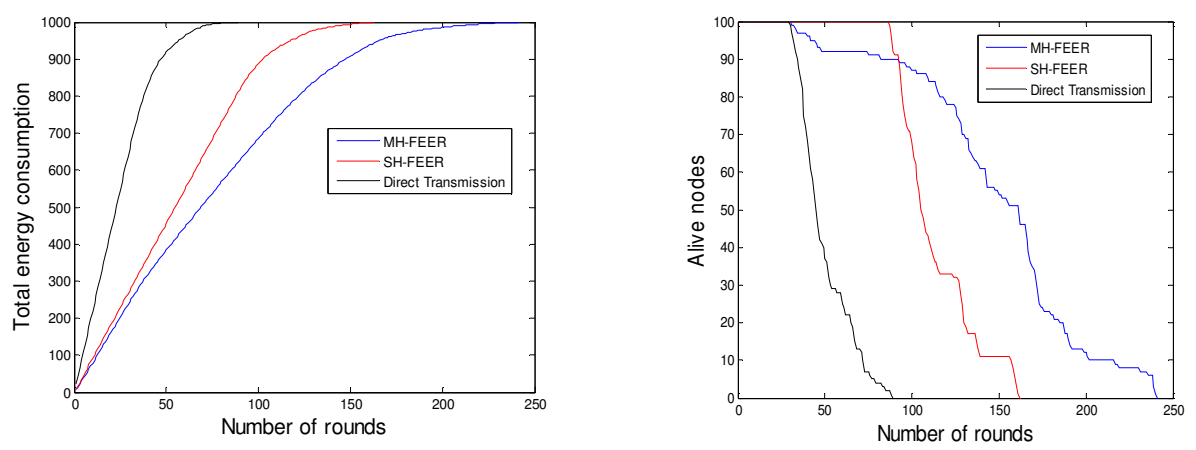

Figure 1. Total energy consumption vs. number of rounds Figure 2. Alive nodes vs. number of rounds

From the simulation result shown in figure 2 and 3, we can see that the first node dies in direct transmission algorithm after 30 rounds while in SH-FEER and MH-FEER first node dies after 87 and 31 rounds respectively. We also observe that the last node dies in direct transmission algorithm after 89 rounds while in SH-FEER and MH-FEER last node dies after 162 and 241 rounds respectively. Therefore, in this set of simulations, we note that MH-FEER about $32.78 \%$ more efficient in term of network lifetime compared to SH-FEER and about $63.07 \%$ than the direct transmission algorithm.

Furthermore, the number of alive nodes decreases speedily with direct transmission and SHFEER cases in comparing to the MH-FEER algorithm. 


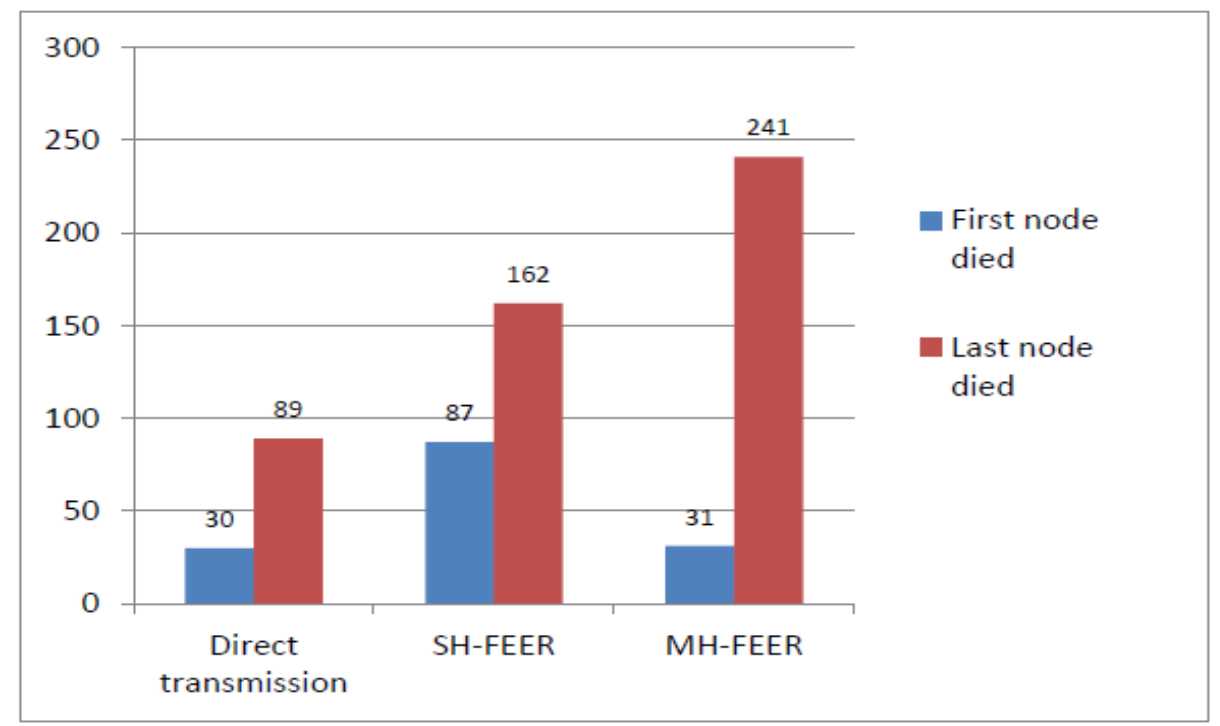

Figure 3. The first and last node died in static topology

\subsection{Simulation 2: dynamic topology}

On account of the environment's impact, underwater sensor nodes will be dynamic, which produces changes in topology. Consequently, to will be more close to reality we must introduce the movement to the simulation conditions. Considering this situation, we have used a random walk mobility model, the node mobility is taken as $1 \mathrm{~m} / \mathrm{s}$ and the other environmental parameters are similar with the static simulation.

For the same number of nodes in the network, the figure 4 also shows that the total energy consumption in dynamic networks is significantly high comparing to the static networks. Furthermore, the number of alive nodes decreases speedily comparing to the static cases $(29.37 \%)$.
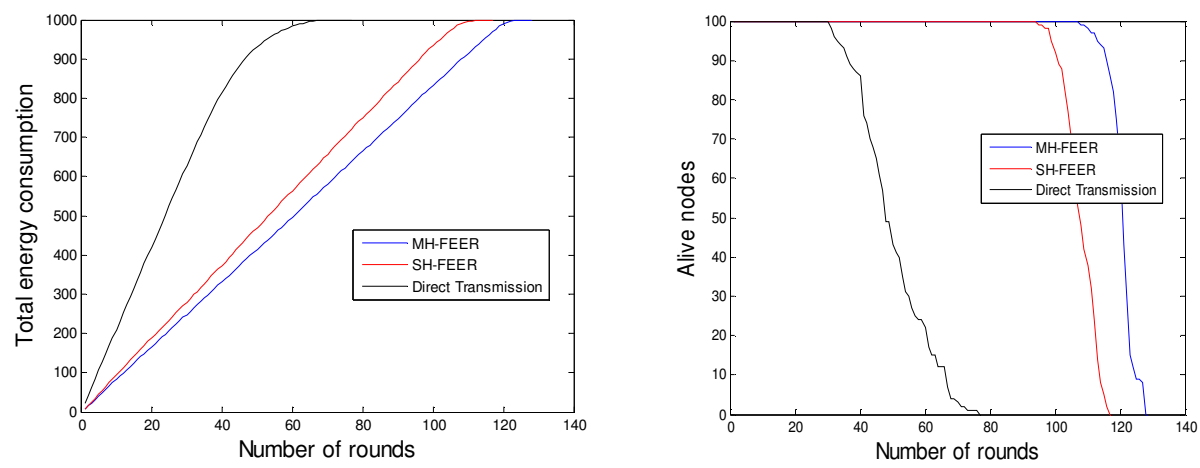

Figure 4. Total energy consumption vs. number of rounds Figure 5. Alive nodes vs. number of rounds 
From the simulation result shown in figure 5 and 6, we can see that the first node dies in direct transmission algorithm after 30 rounds while in SH-FEER and MH-FEER first node dies after 96 and 108 rounds respectively. We also observe that the last node dies in direct transmission algorithm after 77 rounds while in SH-FEER and MH-FEER last node dies after 117 and 128 rounds respectively. Therefore, in this set of simulation we note that MH-FEER about $8.5 \%$ is more efficient in term of network lifetime comparing to SH-FEER and about $39.84 \%$ versus to the direct transmission algorithm.

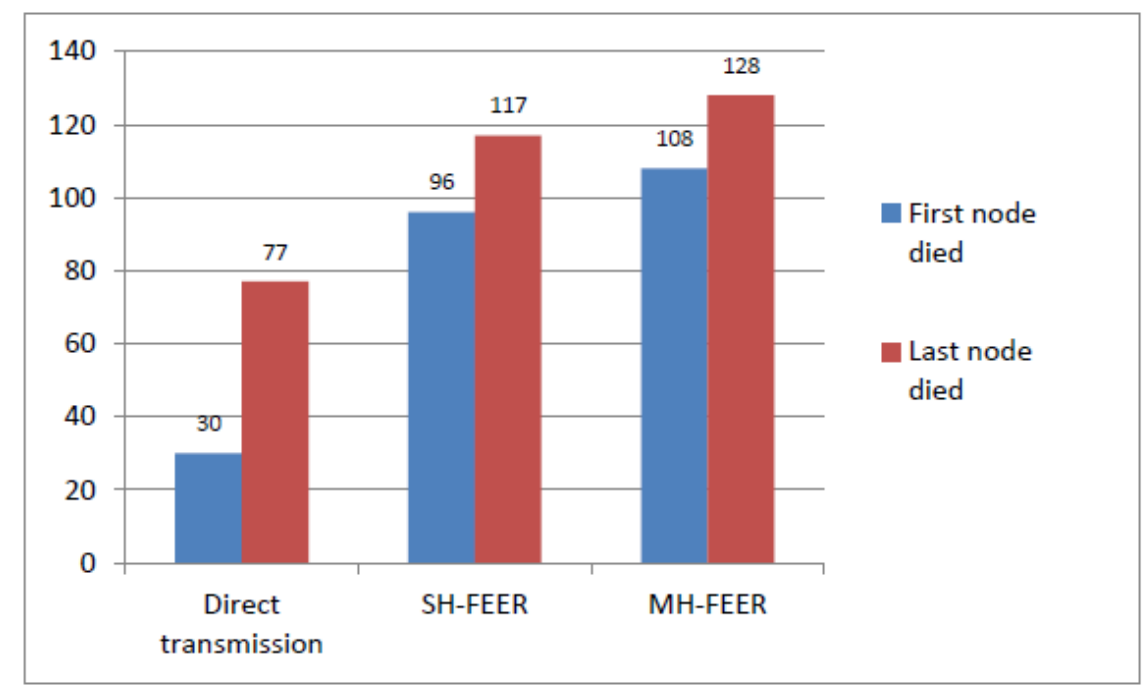

Figure 6. The first and last node died in dynamic topology

\subsection{Impact of density and mobility}

In order to check the effect of density and mobility we choose the second proposition MH-FEER in this set of simulations where all nodes are mobile with the same speed and we change the number of nodes from 200 to 1000 nodes, the simulations results are plotted in the following figures.

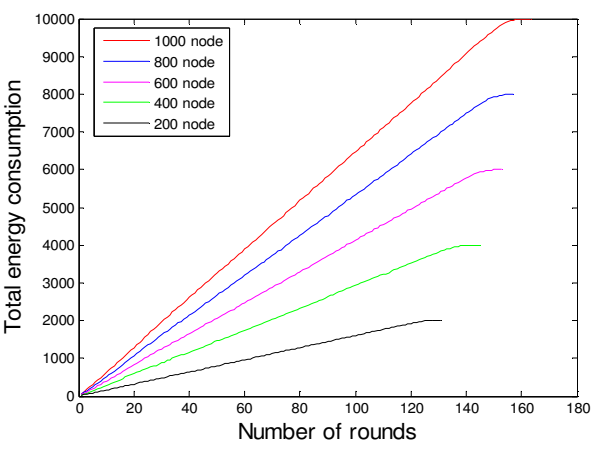

Figure 7. Impact of density on energy consumption

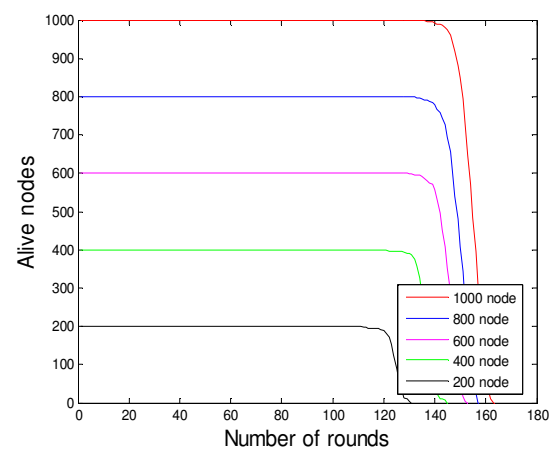

Figure 8. Impact of density on alive node

Figures 7 and 8 depict the impact of density on the total energy consumption and the alive node respectively. The total energy consumption increases when the number of nodes increases since 
more nodes are involved in packet transmission. However the number of alive nodes remains stable but it's eventually decreased rapidly.

In this section of simulation we vary the mobility speed of each node from $1.5 \mathrm{~m} / \mathrm{s}$ to $5.5 \mathrm{~m} / \mathrm{s}$ when using 100 nodes.
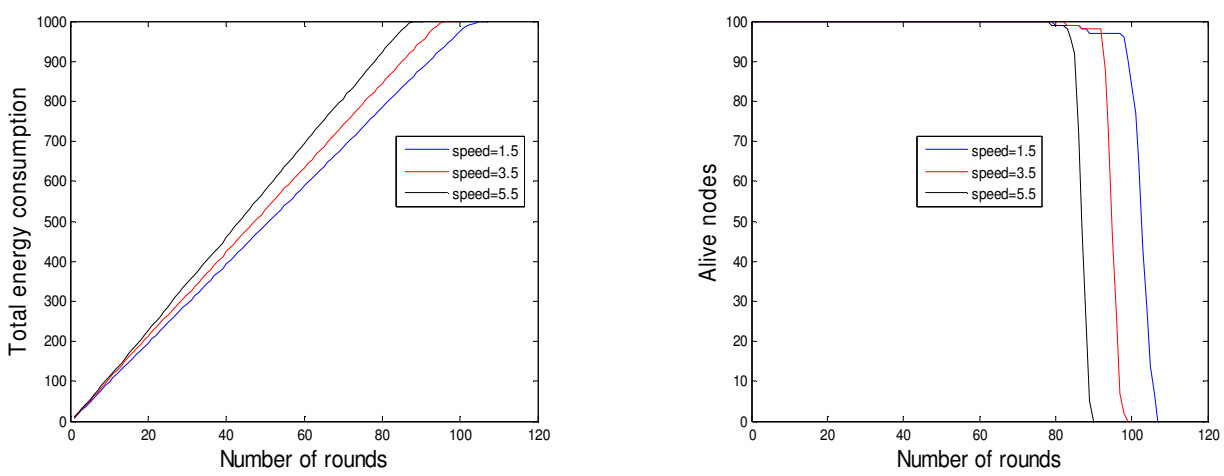

Figure 9.Impact of mobility on energy consumption Figure 10. Impact of mobility on alive nodes

It can be seen from figure 9 that the total energy consumption increases with the growth of the node speed, however, the number of alive nodes reduces more when increasing the speed as illustrated in figure 10.

\section{CONCLUSION}

In this paper we address the issue of routing energy efficiency in underwater wireless sensor networks. Firstly, we have proposed two algorithms which utilize a clustering method based on Fuzzy C-Means, using these algorithms; we study the effect of two key parameters (scalability and mobility) on the performance of UWSN. Moreover theses approaches are deployed on static and mobile environments. The simulation results show a promising performance, in terms of energy consumption and network lifetime, with the proposed SH-FEER and MH-FEER algorithms, than the direct transmission. In the future works we aim to investigate intelligent algorithms such as genetic algorithms or ant colony, specially to find the shortest path between the cluster heads and the underwater sink. 


\section{REFERENCES}

[1] I.F. Akyildiz, D. Pompili, and T. Melodia, "Underwater acoustic sensor networks: Research challenges", Ad Hoc Networks, pp. 257-279, 2005.

[2] L. Liu, S. Zhou, and J.H .Cui, "Prospects and Problems of Wireless Communication for Underwater Sensor Networks", WILEY WCMC, pp. 977-994, 2008.

[3] Z.J. Haas, J.Y. Halpern and L. Li, "Gossip-Based Ad Hoc Routing", Proceedings of the 19th Conference of the IEEE Communications Society (INFOCOM), pp. 1707-1716, 2002.

[4] J. Kulik, W.R. Heinzelman and H. Balakrishnan, "Negotiation based protocols for disseminating information in wireless sensor networks", Wirel.Netw, pp. 169-185, 2002.

[5] C. Intanagonwiwat, R. Govindan, D. Estrin and J.Heidemann, "Directed diffusion for wireless sensor networking", IEEE/ACM Trans.Netw, pp. 2-16, 2003.

[6] Y. Xiu, J. Heidemann, and D. Estrin, "Geography-informed energy conservation for ad-hoc routing", Proceedings ACM/IEEE MobiCom'01, pp. 70-84, 2001.

[7] Y. Yu, R. Govindan, and D.Estrin, "Geographical and energy aware routing: A recursive data dissemination protocol for wireless sensor networks", Technical Report UCLA/CSD-TR-01-0023, UCLA Computer Science Department, 2001.

[8] W.R. Heinzelman, A. Chandrakasan, H. Balakrishnan, "Energy-Efficient Communication Protocol for Wireless Microsensor Networks", In Proceedings of the 33rd Annual Hawaii International Conference on System Sciences, pp. 10-19, 2000.

[9] W.B. Heinzelman, A.P. Chandrakasan, H. Balakrishnan, "An application-specific protocol architecture for wireless microsensor networks", Wireless Commun, pp. 660-670, 2002.

[10] A.B. Dogar, G.A. Shah and M.O. Farooq, "MR-LEACH: Multi-hop routing with low energy adaptive clustering hierarchy", Fourth International Conference on Sensor Technologies and Applications (SENSORCOMM), pp. 262-268, 2010.

[11] O. Younis, S. Fahmy, "A hybrid energy-efficient, distributed clustering approach for ad hoc sensor networks", Mobile Comput, IEEE Trans, pp. 366-79, 2004.

[12] M.C. Domingo, R. Prior, "A distributed clustering scheme for underwater wireless sensor networks. in personal, indoor and mobile radio communications", Proceedings of the IEEE 18th International Symposium on PIMRC,2007.

[13] W. Pu, L. Cheng and Z. Jun, "Distributed minimum-cost clustering protocol for underwater sensor networks (UWSNs) ", Proceedings of the IEEE international conference on communications, ICC '07, 2007.

[14] L. Uichin, et al., "Pressure routing for underwater sensor networks", Proceedings of the IEEE INFOCOM, 2010.

[15] M. Zhang, J. Yu, "Fuzzy partitional clustering algorithms", J journal of Software, pp.6-859, 2004.

[16] M. Hadjila, H. Guyennet and M. Feham, "Energy-Efficient in Wireless Sensor Networks using Fuzzy C-Means Clustering Approach", International Journal of Sensors and Sensor Networks, pp. 21-26, 2013.

[17] E. Sozer, M. Stojanovic, and J. Proakis, "Underwater acoustic networks", IEEE Journal of Oceanic Engineering, pp. 72-83, 2000. 